\title{
Exact SDP relaxations of quadratically constrained quadratic programs with forest structures
}

\author{
Godai Azuma* $\quad$ Mituhiro Fukuda ${ }^{\dagger} \quad$ Sunyoung Kim ${ }^{\ddagger} \quad$ Makoto Yamashita $^{\S}$
}

September, 2020

\begin{abstract}
We study the exactness of the semidefinite programming (SDP) relaxation of quadratically constrained quadratic programs (QCQPs). With the aggregate sparsity matrix from the data matrices of a QCQP with $n$ variables, the rank and positive semidefiniteness of the matrix are examined. We prove that if the rank of the aggregate sparsity matrix is not less than $n-1$ and the matrix remains positive semidefinite after replacing some off-diagonal nonzero elements with zeros, then the standard SDP relaxation provides an exact optimal solution for the QCQP under feasibility assumptions. In particular, we demonstrate that QCQPs with forest-structured aggregate sparsity matrix, such as the tridiagonal or arrow-type matrix, satisfy the exactness condition on the rank. The exactness is attained by considering the feasibility of the dual SDP relaxation, the strong duality of SDPs, and a sequence of QCQPs with perturbed objective functions, under the assumption that the feasible region is compact. We generalize our result for a wider class of QCQPs by applying simultaneous tridiagonalization on the data matrices. Moreover, simultaneous tridiagonalization is applied to a matrix pencil so that QCQPs with two constraints can be solved exactly by the SDP relaxation.
\end{abstract}

Key words. Quadratically constrained quadratic programs, exact semidefinite relaxations, forest graph, the rank of aggregated sparsity matrix.

AMS Classification. 90C20, 90C22, 90C25, 90C26.

\section{Introduction}

We consider a quadratically constrained quadratic program (QCQP):

$$
\begin{array}{ll}
\text { minimize } & x^{\mathrm{T}} Q^{0} x+2 q_{0}{ }^{\mathrm{T}} x \\
\text { subject to } & x^{\mathrm{T}} Q^{p} x+2 q_{p}{ }^{\mathrm{T}} x \leq b_{p}, \quad p=1, \ldots, m,
\end{array}
$$

\footnotetext{
*Department of Mathematical and Computing Science, Tokyo Institute of Technology, 2-12-1-W8-29 OhOkayama, Meguro-ku, Tokyo 152-8552, Japan.

${ }^{\dagger}$ Department of Mathematical and Computing Science, Tokyo Institute of Technology, 2-12-1-W8-41 OhOkayama, Meguro-ku, Tokyo 152-8552, Japan (mituhiro@is.titech.ac.jp).

${ }^{\ddagger}$ Department of Mathematics, Ewha W. University, 52 Ewhayeodae-gil, Sudaemoon-gu, Seoul 03760, Korea (skim@ewha.ac.kr). The research was supported by NRF 2017-R1A2B2005119.

${ }^{\S}$ Department of Mathematical and Computing Science, Tokyo Institute of Technology, 2-12-1-W8-29 OhOkayama, Meguro-ku, Tokyo 152-8552, Japan (Makoto.Yamashita@c.titech.ac.jp). This research was partially supported by JSPS KAKENHI (Grant number: 20H04145).
} 
where $Q^{p} \in \mathbb{S}^{n}, q^{p} \in \mathbb{R}^{n}$, and $b_{p} \in \mathbb{R}$ for $p=0,1, \ldots, m$ are problem data and $x \in \mathbb{R}^{n}$ is the variable. Nonconvex QCQPs of the form (11) arise from a wide range of applications, for instance, sensor network localization problems [5], quadratic assignment problems [17, 25], equally deployment problems [26, 27, and optimal power flow problems [6, 19, 35]. As some NP-hard problems can be reformulated by QCQPs, they are known to be NP-hard in general. Nonconvex QCQPs are approximately solved with tractable convex relaxations such as semidefinite programming (SDP) relaxations.

SDP relaxations of QCQPs are regarded as a powerful convex relaxation that provides tight lower bounds for (11) 22]. Both theoretical and computational aspects of SDP relaxations [2, 4, 6, 7, 15, 22, 31, 32, 33, 35] have been extensively studied. For QCQPs in the form of (11), by letting $X=x x^{\mathrm{T}}$ and relaxing it to $X-x x^{\mathrm{T}} \succeq O$, we have the standard SDP relaxation as

$$
\begin{array}{cl}
\operatorname{minimize} & Q^{0} \bullet X+2 q_{0}{ }^{\mathrm{T}} x \\
\text { subject to } & Q^{p} \bullet X+2 q_{p}{ }^{\mathrm{T}} x \leq b_{p}, \quad p=1, \ldots, m, \\
X \succeq x x^{\mathrm{T}} &
\end{array}
$$

where $Q^{p} \bullet X$ denotes the Frobenius inner product of $Q^{p}$ with $X$, and $X \succeq x x^{\mathrm{T}}$ denotes that $X-x x^{\mathrm{T}}$ is positive semidefinite. Computational studies on the SDP relaxation (2) for an approximate lower bound of (1) have been focused on improving the computational efficiency of solution methods. Primal-dual interior-point methods or bundle's methods are some of widely used computational methods to solve large-sized SDP relaxations [33. In particular, the aggregate sparsity of data matrices has been successfully used to reduce the size of the SDP relaxation when imploying primal-dual interior-point methods [9, 16, 21]. Recently, more efficient algorithms based on the first-order methods, for instance, SDPNAL+ 34] and BBCPOP [11], have been introduced for large-scale QCQPs.

For the theoretical study on the SDP relaxation, the rank of the SDP solution plays an important role. As the feasible set of the SDP relaxation is larger than that of the original QCQP in general, an approximate solution to nonconvex QCQP (1) is usually obtained by solving the SDP relaxation (2). The rank of the SDP solution can be determined after or prior to solving (2). If the rank of the computed SDP solution is one, or the matrix $\left[1,\left(x^{*}\right)^{\mathrm{T}} ; x^{*}, X^{*}\right]$ is rank-1, then the SDP relaxation is called exact. With the rank-1 SDP solution, $x^{*}$ satisfying $X^{*}=x^{*} x^{* \mathrm{~T}}$ recovers the relaxed constraint. For some class of QCQPs, however, the rank of the SDP solution is known prior to solving (2). QCQPs with nonpositive off-diagonal data matrices were known to be solved exactly by the SDP relaxation in [6, 15, 19]. In particular, the exactness of the SDP relaxation for QCQPs with complex variables associated with connected and acyclic graphs was studied in [6] where some sign properties of $Q^{p}(p=1, \ldots, m)$ were assumed. Low rank SDP solutions and the upper bounds for the rank of the SDP solution were also studied by Pataki [23], Laurent and Vavitsiotis [18], and Madani et al. [20].

Recently, Burer and Ye in [7] proposed a method to determine the rank of SDP solutions for some class of QCQPs prior to solving the SDP relaxation. For diagonal QCQPs with diagonal $Q^{0}, Q^{1}, \ldots, Q^{m}$, they showed that the rank of SDP solutions is bounded above by $n-f+1$, where the feasibility number $f$ is determined by considering the systems for $j=1, \ldots, n$ :

$$
\begin{aligned}
& Q^{0} \bullet X+\left[q_{0}\right]_{j} x_{j}=-1, \\
& Q^{i} \bullet X+\left[q_{i}\right]_{j} x_{j} \leq 0, \quad \forall i=1, \ldots, m, \\
& X: \text { diagonal, } \quad X_{k k} \geq 0, \quad \forall k \neq j .
\end{aligned}
$$


More precisely, $f=\mid\{j \mid$ the above system with $j$ is feasible, $1 \leq j \leq n\} \mid$. Some exactness conditions for the diagonal QCQPs were provided by analyzing the case where the upper bound $n-f+1$ equals one, and their result was extended to random or non-random QCQPs. More recently, Wang and Kilinç-Karzan 32 analyzed the faces of the convex Lagrangian multipliers $\Gamma$ of the SDP relaxation for a QCQP. They stablished conditions for which the exact SDP relaxation holds and, in particular, it includes the result of [7] for diagonal QCQPs.

Special classes of QCQPs that admit the exact SDP relaxation has also been studied. For instance, the Generalized Trust-Region Subproblem (GTRS) that minimizes a quadratic objective over a quadratic constraint is such a class. The GTRS is, in fact, a QCQP (1) with only one constraint $(m=1)$. It generalizes the classical Trust-Region Subproblem (TRS) in which a quadratic objective is minimized over a Euclidean ball. Since the objective of the TRS is allowed to be nonconvex, the TRS is nonlinear and nonconvex; however, its SDP relaxation is always exact. The GTRS shares nice properties with the TRS. In fact, under the Slater's condition due to the S-lemma [24], the GTRS admits an exact SDP relaxation. Generalized eigenvalue problems are closely related to the GTRS. Adachi and Nakatsukasa in [1 developed an eigenvalue-based algorithm for the GTRS. Recently, Wang and Kilinç-Karzan 31] analyzed the convex hull of a nonconvex feasible set using the generalized eigenvalue of a matrix pencil $Q^{0}-\lambda Q^{1}$ which is also used in subsection 4.2 of this paper.

The main purpose of this paper is to present sufficient conditions for the SDP relaxation to be exact for some classes of QCQPs, considering the aggregate sparsity matrix of data matrices $Q^{0}, Q^{1}, \ldots, Q^{m}$. We assume that the aggregate sparsity matrix is positive semidefinite in addition to mild feasibility assumptions. We show that if (i) the rank of the aggregate sparsity matrix is not less than $n-1$ for any nonzero values of the matrix and (ii) the positive semidefiniteness of the matrix is maintained even when some of off-diagonal elements of the matrix become zeros; then the SDP relaxation of the QCQPs is exact. The aggregate sparsity matrices satisfying (i) and (ii) focused in this paper are the matrices associated to forest-structured graphs such as tridiagonal and arrow-type matrix. We call QCQPs (11) forest-structured QCQPs, tridiagonal QCQPs, or arrowtype QCQPs if the indices of maximal cliques obtained from the aggregate sparsity matrix form a "forest", a tridiagonal matrix, or an arrow-type matrix, respectively, sometimes with permutation. These classes of QCQPs admit the exact SDP relaxation under some assumptions on the feasible set of QCQPs. We also extend our results on tridiagonal QCQPs to general QCQPs via simultaneous tridiagonalization.

The aggregate sparsity matrix from the data matrices of QCQPs in this paper is employed to examine the rank of the SDP dual solution, while it has been mostly studied for improving computational efficiency of solving the SDP relaxation [9, 16, 21, 28, Moreover, our results show that the exact SDP relaxation can be proved for forest-structured QCQPs, regardless of signs of data matrices [6, 15, 30. Note that Bose et al. 6] also considered connected and acyclic graphs associated with the aggregate sparsity matrices. For the classes of forest-structured QCQPs, including tridiagonal or arrow-type QCQPs, the second-order cone relaxation also provides the exact optimal solution [15]. We also note that the exactness conditions by Burer and Ye [7] cannot be used for determining the exactness of the SDP relaxation for tridiagonal QCQPs since diagonal QCQPs in [7] is a special case of tridiagonal QCQPs.

For tridiagonal QCQPs, we consider at most $n-1$ systems corresponding to the elements of the positive semidefinite variable matrix in the dual SDP relaxation, which is described as $\left(\mathcal{F}_{k \ell}\right)$ in section 3. Each system consists of constraints in the dual SDP relaxation of (2) with $q_{p}=0$ for $p=0,1, \ldots, m$ and the constraint that an element of the positive semidefinite variable matrix should be zero. If the system has no solutions for all $(k, \ell) \in\{(i, i+1) \mid i=1, \ldots, n-1\}$, then 
we show that the SDP relaxation is exact. More precisely, our condition on the exactness is that the system $\left(\overrightarrow{\left.\mathcal{F}_{k \ell}\right)}\right.$ is not feasible for all $(k, \ell) \in\{(i, i+1) \mid i=1, \ldots, n-1\}$. Since the number of elements in $\{(i, i+1) \mid i=1, \ldots, n-1\}$ is $n-1$, we can determine whether the SDP relaxation of a given tridiagonal QCQP is exact by considering at most $n-1$ systems. The exactness of the SDP relaxation for other forest-structured QCQPs can be discussed similarly.

To prove the exactness of the SDP relaxation with our sufficient conditions for a foreststructured QCQP, the rank property on the aggregated sparsity matrix in Lemma 2.2 and the perturbation technique in Lemma 3.3 are utilized. We need to estimate a lower bound for the rank of the dual SDP as in Burer and Ye's work [7]. The main idea there was to estimate the upper bound for the rank of a SDP solution $\left(x^{*}, X^{*}\right)$ by the lower bound for the rank for a dual solution $y^{*}$ of the dual SDP. The strong duality of SDP and the Sylvester's inequality on the rank were used in [7]. In this work, a lower bound for the rank is estimated using the lower bound for the rank $n-1$ for a forest-structured QCQP with $n$ variables and nonzero off-diagonal elements.

We also investigate the exactness of the SDP relaxation for non-tridiagonal QCQPs by applying our results on tridiagonal QCQPs. For instance, most GTRS's are not tridiagonal QCQPs. However, the exactness of the SDP relaxation for the GTRS was known by the S-lemma [24] under the Slater's conditions. We demonstrate that the GTRS has the exact SDP relaxation without replying on the S-lemma. More precisely, we show the exactness of the SDP relaxation for the GTRS by our result on tridiagonal QCQPs. To apply the results on tridiagonal QCQPs, the GTRS should be transformed to the tridiagonal QCQP. We improve the simultaneous tridiagonalization technique proposed in [29] so that all tridiagonal QCQPs constructed from the GTRS always satisfy the conditions for the exact SDP relaxation. Similarly, the exactness of other classes of QCQPs can be analyzed by the method presented in this paper.

The rest of this paper is organized as follows. In section 2, we review related works on the exact SDP relaxation of QCQPs, and present some background materials for the subsequent discussion. Some basic properties on tridiagonal matrices are also summarized to discuss on tridiagonal QCQPs. Sections 3 and 4 include the main results of this paper. In section 3, the main results for forest-structured QCQPs are described, and sufficient conditions for the exactness are also proposed. A perturbation technique used in the proofs for these conditions is also shown. In section 4, we first describe the simultaneous tridiagonalization technique. Then, the conditions presented in section 3 are applied to non-tridiagonal QCQPs. We also present an alternative proof on the exactness of Generalized Trust-Region Subproblem in section 4. Finally, we conclude in section 5.

\section{Preliminaries}

We start by introducing notation and symbols used in this paper.

\section{$2.1 \quad$ Notation and symbols}

- $\mathbb{R}^{n}$ and $\mathbb{S}^{n}$ denote the $n$-dimensional Euclidean space, the space of $n \times n$ symmetric matrices, respectively. The notation $M \succeq O$ and $M \succ O$ mean that the matrix $M$ is positive semidefinite and positive definite, respectively.

- $0_{n} \in \mathbb{R}^{n}$ denotes the zero vector of length $n$. $I_{n} \in \mathbb{R}^{n \times n}$ denotes the $n \times n$ identity matrix.

- For $M, N \in \mathbb{S}^{n}, M \bullet N$ denotes the Frobenius inner product of $M$ and $N$, i.e., $M \bullet N:=$ $\operatorname{tr}\left(M^{\mathrm{T}} N\right)=\sum_{i, j} M_{i j} N_{i j}$. 
- For $M \in \mathbb{S}^{n},\|M\|_{\max }$ denotes the maximum norm of $M$, i.e., $\|M\|_{\max }=\max _{1 \leq i, j \leq n}\left|M_{i j}\right|$.

- $[n]$ is a shorthand notation for $\{i \in \mathbb{N} \mid 1 \leq i \leq n\}$.

- $M_{I J}$ denotes the submatrix of $M$ constructed by collecting the rows of $M$ indexed by $I \subset[n]$ and the columns of $M$ indexed by $J \subset[n]$. We use $M_{I}$ for $M_{I I}$.

Let $M=\left[m_{i j}\right] \in \mathbb{S}^{n}$ for $1 \leq i, j \leq n$. We use $\left[m_{i j}\right]$ to denote a matrix $M \in \mathbb{S}^{n}$ whose $(i, j)$-th element is $m_{i j}$ and also use $\left[Q^{0}\right]_{i j}$ to denote the $(i, j)$-th element of a matrix $Q^{0} \in \mathbb{S}^{n}$.

Definition 2.1. A finite set $\left\{a_{1}, \ldots, a_{n}\right\} \subset \mathbb{R}$ is called sign-definite with respect to $\mathbb{R}$ if its members are either all nonnegative or all nonpositive, i.e., $a_{i} a_{j} \geq 0$ for any $i, j \in[n]$.

For example, the set $\{0,100,0,2\}$ is sign-definite while sets $\{0,100,0,-2\}$ and $\{-1,1\}$ are not sign-definite.

\subsection{Tridiagonal matrices}

A matrix $M=\left[m_{i j}\right] \in \mathbb{S}^{n}$ is called tridiagonal if all elements $m_{i j}$ are zero for $i, j \in[n]$ satisfying $|i-j| \geq 2$. We use $\mathrm{d}(n)$ and $\operatorname{od}(n)$ to represent the index sets for the main diagonal and off-diagonal elements of $n \times n$ matrices, respectively, i.e.,

$$
\begin{aligned}
\mathrm{d}(n) & :=\{(i, i) \mid i=1, \ldots, n\}, \\
\operatorname{od}(n) & :=\{(i, i+1) \mid i=1, \ldots, n-1\} .
\end{aligned}
$$

Since $M \in \mathbb{S}^{n}, \operatorname{od}(n)$ contains only the indices for the upper triangular elements.

We discuss a method to estimate a lower bound on the rank of tridiagonal matrices. For general matrices, this estimation is generally hard. In the case of a diagonal matrix, we know that its rank equals the number of nonzero elements on its main diagonal. For a tridiagonal matrix, we can show that its rank can be bounded from below by the number of off-diagonal nonzero elements. The following lemma is immediately obtained from the result of [12].

Lemma 2.2. Let $M \in \mathbb{S}^{n}$ be a tridiagonal matrix. If all the superdiagonal elements of $M$ are nonzeros, then $\operatorname{rank} M \geq n-1$.

For symmetric positive semidefinite matrices $\mathbb{S}^{n} \ni M \succeq O$, it is difficult to determine whether the positive semidefiniteness is maintained after replacing some of off-diagonal elements with zeros. However, in the case of positive semidefinite tridiagonal matrices, we show in the following lemma that they remain to be positive semidefinite even if some of off-diagonal elements are replaced by zeros.

Lemma 2.3. Let $M=\left[m_{i j}\right] \in \mathbb{S}^{n}$ be a positive semidefinite and tridiagonal matrix. For a subset $E \subset \operatorname{od}(n)$, let $L=\left[\ell_{i j}\right] \in \mathbb{S}^{n}$ be the tridiagonal matrix constructed by replacing the matrix elements of $M$ indexed by $E$ with zeros, i.e.,

$$
\ell_{i j}:= \begin{cases}0 & \text { if }(i, j) \in E \text { or }(j, i) \in E \\ m_{i j} & \text { otherwise }\end{cases}
$$

Then, $L \succeq O$. 
Proof. We use induction on the size of the set $E$. Let us first consider the case $|E|=1$. Then, $E$ has one element $(i, i+1) \in E$ for some $i$. For any $I \subset[n]$, we have the following two cases:

(a) If $i \notin I$ or $i+1 \notin I$, the principal $L_{I}$ does not have $(i, i+1)$-th and $(i+1, i)$-th elements of $M$, therefore

$$
\operatorname{det}\left(L_{I}\right)=\operatorname{det}\left(M_{I}\right) \geq 0 \text {. }
$$

(b) If $i \in I$ and $i+1 \in I$, the principal $L_{I}$ has $(i, i+1)$-th and $(i+1, i)$-th elements of $M$ replaced by zeros. Since the submatrix $L_{I}$ is a block diagonal matrix with two blocks,

$$
\begin{aligned}
\operatorname{det}\left(L_{I}\right) & =\operatorname{det}\left(L_{I \cap\{1, \ldots, i\}}\right) \operatorname{det}\left(L_{I \cap\{i+1, \ldots, n\}}\right) \\
& =\operatorname{det}\left(M_{I \cap\{1, \ldots, i\}}\right) \operatorname{det}\left(M_{I \cap\{i+1, \ldots, n\}}\right) \\
& \geq 0,
\end{aligned}
$$

where the last inequality follows from the fact that all the principal minors of $M$ are nonnegative.

Since all the principal minors of $L$ are nonnegative, $L \succeq O$ follows.

Suppose the result is true for $|E|=k-1$, and consider the case $|E|=k$. The set $E$ can be divided into two sets: $F:=\{(j, j+1)\}$ and $E \backslash F$. Let $N \in \mathbb{S}^{n}$ be a tridiagonal matrix constructed by replacing the $(j, j+1)$ and $(j+1, j)$ elements of $M$ with zeros. Then, from the case mentioned above, $N \succeq O$ holds. Since $|E \backslash F|=k-1$, by the induction hypothesis, we have $L \succeq O$.

In our proof of Lemma 2.3, the nonnegativeness of all the principal minors of $L$ was shown based on the fact that they are given by the product of at most two principal minors of $M$. The nonnegativeness can be also shown by a representation of the determinant of the tridiagonal matrix. More details can be found in Corollary 2.2 of [8].

\subsection{Aggregate sparsity matrix and forest-structured QCQPs}

In Lemma 2.2, the rank of tridiagonal matrices is discussed without taking the positive semidefiniteness into account. As the rank of positive semidefinite matrices plays a crucial role to extend the result in Lemma 2.2 to more general matrices, we briefly introduce the aggregate sparsity matrix and discuss the relation between the aggregate sparsity matrix and forest-structured QCQPs.

To construct the aggregate sparsity matrix from the SDP relaxation (2), we define an aggregate sparsity graph $G(\mathcal{V}, \mathcal{E})$ as a graph with the set of vertices $\mathcal{V}=[n]$ and the set of edges

$$
\mathcal{E}=\left\{(i, j) \in \mathcal{V} \times \mathcal{V} \mid\left[Q^{p}\right]_{i j} \neq 0 \text { for some } p \in\{0\} \cup[m]\right\} .
$$

The sparsity encoded in $\mathcal{E}$ is called the aggregate sparsity pattern. The aggregate sparsity matrix $R \in \mathbb{S}^{n}$ corresponding to $G(\mathcal{V}, \mathcal{E})$ is defined as

$$
R_{i j}= \begin{cases}* & \text { if }(i, j) \in \mathcal{E} \\ 0 & \text { otherwise }\end{cases}
$$

where $*$ is an arbitrary nonzero real number.

A graph is called a forest if it has no cycles in the upper triangular part $\mathcal{E} \cap\{(i, j) \in \mathcal{V} \times \mathcal{V} \mid i<j\}$. A connected forest is called a tree. We also call QCQPs (10) as forest-structured QCQPs if their 
aggregated sparsity graphs $G(\mathcal{V}, \mathcal{E})$ are forests. Thus, the graph $G(\mathcal{V}, \mathcal{E})$ obtained from foreststructured QCQPs consists of one or more trees. Obviously, the tridiagonal QCQP is a subclass of forest-structured QCQP.

For a given symmetric matrix $M=\left[m_{i j}\right] \in \mathbb{S}^{n}$, a sparsity structure graph $G(\mathcal{V}, \mathcal{E})$ can also be defined as an graph with the set of vertices $\mathcal{V}=[n]$ and the set of edges

$$
\mathcal{E}=\left\{(i, j) \in \mathcal{V} \times \mathcal{V} \mid m_{i j} \neq 0\right\} .
$$

We call symmetric matrices $M \in \mathbb{S}^{n}$ as forest-structured matrices if their sparsity structure graphs have no cycles. For $G(\mathcal{V}, \mathcal{E})$ corresponding to a QCQP, the sparsity structure graph of $Q^{p}$ is a subgraph of $G(\mathcal{V}, \mathcal{E})$ for any $p \in\{0\} \cup[m]$. As a result, all the matrices $Q^{p}(p \in\{0\} \cup[m])$ in forest-structured QCQPs must be forest-structured matrices.

We consider the following index sets in the subsequent discussion for the forest-structured matrices and QCQPs:

$$
\begin{aligned}
\mathrm{d}(n) & :=\{(i, i) \mid i=1, \ldots, n\} \\
\operatorname{od}(n) & :=\{(i, j) \in \mathcal{E} \mid i<j\},
\end{aligned}
$$

where $(i, j)$ is the index for the nonzero element of the aggregate sparsity matrix. By an appropriate permutation on $[n]$, we can assume that $n \in \mathcal{V}$ is the root of a tree graph. If $j$ corresponds to the parent node of $i$ in a tree graph, then it is uniquely determined for each $i \in \mathcal{V} \backslash\{n\}$.

As an immediate consequence of Corollary 3.9 of [14, we can establish that any symmetric positive semidefinite matrix whose graph is a tree has rank at least $n-1$, as described in the following lemma. We will use this fact to estimate the rank of SDP solutions of SDP relaxations in section 3 ,

Lemma 2.4. [14, Corollary 3.9] Let $M \in \mathbb{S}^{n}$ be a positive semidefinite and forest-structured matrix. If the sparsity structure graph of $M$ is connected and all the off-diagonal elements in $\operatorname{od}(n)$ for $M$ are nonzeros, then $\operatorname{rank} M \geq n-1$.

As an example of forest-structured QCQPs, we consider an arrow-type matrix of form:

$$
V=\left(\begin{array}{ccccc}
v_{1} & & & & w_{1} \\
& v_{2} & & & w_{2} \\
& & \ddots & & \vdots \\
& & & v_{n-1} & w_{n-1} \\
w_{1} & w_{2} & \cdots & w_{n-1} & v_{n}
\end{array}\right) .
$$

For the arrow-type matrix, the index sets $\mathrm{d}(n)$ and $\operatorname{od}(n)$ are given as

$$
\begin{aligned}
\mathrm{d}(n) & :=\{(i, i) \mid i=1, \ldots, n\} \\
\operatorname{od}(n) & :=\{(i, n) \mid i=1, \ldots, n-1\} .
\end{aligned}
$$

By Lemma 2.4, if $V \succeq O$ and $w_{1}, \ldots, w_{n-1}$ are nonzeros, then the rank of $V$ is at least $n-1$.

Lemma 2.3 can also be extended to forest-structured matrices.

Lemma 2.5. Let $M=\left[m_{i j}\right] \in \mathbb{S}^{n}$ be a positive semidefinite and forest-structured matrix. For a subset $E \subset \operatorname{od}(n)$, let $L=\left[\ell_{i j}\right] \in \mathbb{S}^{n}$ be the forest-structured matrix constructed by replacing the matrix elements of $M$ indexed by $E$ with zero, i.e.,

$$
\ell_{i j}:= \begin{cases}0 & \text { if }(i, j) \in E \text { or }(j, i) \in E \\ m_{i j} & \text { otherwise }\end{cases}
$$

Then, $L \succeq O$. 
Proof. It suffices to consider the case $|E|=1$ since similar arguments to the proof of Lemma 2.3 can be applied. In this case, there exists only one element $(i, j)$ in $E$. By removing the edge $(i, j)$ from $G(\mathcal{V}, \mathcal{E})$ of the forest-structured matrix $M$, a tree in $G(\mathcal{V}, \mathcal{E})$ is divided into two trees: one with the node $i$, and the other with the node $j$. The set $\mathcal{V}$ is also separated into two sets: $W_{1} \subset \mathcal{V}$, the set of nodes in the component including the node $i$ in the graph $G(\mathcal{V}, \mathcal{E} \backslash\{(i, j),(j, i)\})$, and $W_{2} \subset \mathcal{V}$, the set of other nodes. Without loss of generality, we may assume that the indices in $W_{1}$ and $W_{2}$ are consecutive integers, i.e., there exists a positive number $\ell$ such that $W_{1}=[\ell]$ and $W_{2}=[n] \backslash[\ell]$. For any $I \subset[n]$, we have the following two cases:

(a) If $i \notin I$ or $j \notin I$, the principal $L_{I}$ does not include $(i, j)$-th and $(j, i)$-th elements of $M$, then

$$
\operatorname{det}\left(L_{I}\right)=\operatorname{det}\left(M_{I}\right) \geq 0 .
$$

(b) If $i \in I$ and $j \in I$, the principal $L_{I}$ includes $(i, j)$-th and $(j, i)$-th elements of $M$, and their values are zeros. Since the submatrix $L_{I}$ is a block diagonal matrix with two blocks, we have that

$$
\begin{aligned}
\operatorname{det}\left(L_{I}\right) & =\operatorname{det}\left(L_{I \cap W_{1}}\right) \operatorname{det}\left(L_{I \cap W_{2}}\right) \\
& =\operatorname{det}\left(M_{I \cap W_{1}}\right) \operatorname{det}\left(M_{I \cap W_{2}}\right) \\
& \geq 0
\end{aligned}
$$

where the last inequality follows from the fact that all the principal minors of $M$ are nonnegative.

Since all the principal minors of $L$ are nonnegative, we have $L \succeq O$.

\section{$3 \quad$ Exactness conditions for forest-structured QCQPs}

To state our main results on the exact SDP relaxation of forest-structured QCQPs, some assumptions are necessary. Also, a perturbed QCQP is introduced by slightly varying the elements of the data matrix of the objective function. We then present preliminary results on the perturbed QCQP. By analyzing the conditions under which the SDP relaxation has a rank-1 solution, we discuss the exact SDP relaxation for forest-structured QCQPs.

We assume the following for a given QCQP and its SDP relaxation:

\section{Assumption 3.1.}

(a) There exists a feasible point for (11).

(b) There exists $\bar{y} \geq 0$ satisfying $\sum_{p=1}^{m} \bar{y}_{p} Q^{p} \succ O$.

(c) There exists an interior feasible points for (2).

We note that these assumptions were also used for the diagonal QCQPs to establish the results on the exact SDP relaxations in [7]. Assumption 3.1](b) can be also represented as $\bar{y} \leq 0$ and $\sum_{p=1}^{m} \bar{y}_{p} Q^{p} \prec O$. By Assumption 3.1 (a) and (b)] we see that the feasible sets of (1) and (2) are 
bounded, and a solution to (11) exists. In fact, multiplying $x^{\mathrm{T}} Q^{p} x+2 q_{p}{ }^{\mathrm{T}} x \leq b_{p}$ by $\bar{y}_{p}$, and adding these for $p$, we have

$$
x^{\mathrm{T}}\left(\sum_{p=1}^{m} \bar{y}_{p} Q^{p}\right) x+2\left(\sum_{p=1}^{m} \bar{y}_{p} q_{p}\right)^{\mathrm{T}} x \leq b^{\mathrm{T}} \bar{y} .
$$

Thus, all the feasible points of (1) are in the ellipsoid given by the above inequality. It also implies that all the feasible points of (2) is bounded as:

$$
\left(\sum_{p=1}^{m} \bar{y}_{p} Q^{p}\right) \bullet X+2\left(\sum_{p=1}^{m} \bar{y}_{p} q_{p}\right)^{\mathrm{T}} x \leq b^{\mathrm{T}} \bar{y} .
$$

By Assumption 3.1 (b) and (c), the strong duality holds for the primal SDP (2).

The homogeneous form of (11) can be expressed as the following QCQP with $n+1$ variables and $m+2$ inequality constraints:

$$
\begin{array}{ll}
\text { minimize } & z^{\mathrm{T}} \bar{Q}^{0} z \\
\text { subject to } & z^{\mathrm{T}} \bar{Q}^{p} z \leq b_{p}, \quad p=1, \ldots, m, \\
& z^{\mathrm{T}} E_{11} z \leq 1, \quad-z^{\mathrm{T}} E_{11} z \leq-1,
\end{array}
$$

with variable $z \in \mathbb{R}^{n+1}$ where

$$
\bar{Q}^{p}:=\left[\begin{array}{cc}
0 & q_{p}{ }^{\mathrm{T}} \\
q_{p} & Q^{p}
\end{array}\right]
$$

and $E_{i j}$ is an $n \times n$ matrix given by

$$
\left[E_{i j}\right]_{q r}= \begin{cases}1 & \text { if } i=q \text { and } j=r, \\ 0 & \text { otherwise. }\end{cases}
$$

We note that the homogeneous form does not include the linear terms $q_{p}{ }^{\mathrm{T}} x$ for all $p$, and the objective function and the constraints are in quadratic forms in the variables. By the last two inequalities, $z_{1}=1$ or -1 . Although the homogeneous QCQP has a simpler form than the original (11), any solution $z^{*}$ of the homogeneous QCQP recovers a solution $x^{*}=\left[z_{2}^{*} / z_{1}^{*}, \ldots, z_{n+1}^{*} / z_{1}^{*}\right]^{\mathrm{T}}$ of (11). As a result, we may assume, without loss of generality, the following condition:

\section{Assumption 3.2.}

(d) $q_{0}, q_{1}, \ldots, q_{m}$ are zero vectors.

We now consider a homogeneous QCQP:

$$
\begin{array}{ll}
\operatorname{minimize} & x^{\mathrm{T}} Q^{0} x \\
\text { subject to } & x^{\mathrm{T}} Q^{p} x \leq b_{p}, \quad p=1, \ldots, m .
\end{array}
$$

Then, the SDP relaxation of QCQP (4) is described as

$$
\begin{aligned}
\text { minimize } & Q^{0} \bullet X \\
\text { subject to } & Q^{p} \bullet X \leq b_{p}, \quad p=1, \ldots, m, \\
X & \succeq O,
\end{aligned}
$$


and its dual SDP problem is:

$$
\begin{aligned}
\operatorname{maximize} & -b^{\mathrm{T}} y \\
\text { subject to } \quad S(y):=Q^{0}+\sum_{p=1}^{m} y_{p} Q^{p} & \succeq O, \\
y & \geq 0 .
\end{aligned}
$$

\subsection{Perturbed QCQPs}

To perturb the original QCQP (4), we let $P \in \mathbb{S}^{n}$ be a nonzero matrix, and let $\varepsilon>0$ represent how much perturbation is added to the objective function. When a perturbation $\varepsilon P$ is added to the objective function of (4), we have an $\varepsilon$-perturbed QCQP:

$$
\begin{array}{ll}
\operatorname{minimize} & x^{\mathrm{T}}\left[Q^{0}+\varepsilon P\right] x \\
\text { subject to } & x^{\mathrm{T}} Q^{p} x \leq b_{p}, \quad p=1, \ldots, m .
\end{array}
$$

The SDP relaxation of (7) can be written as:

$$
\begin{array}{cc}
\text { minimize } & {\left[Q^{0}+\varepsilon P\right] \bullet X} \\
\text { subject to } & Q^{p} \bullet X \leq b_{p}, \quad p=1, \ldots, m \\
& X \succeq O .
\end{array}
$$

QCQP (41) and its $\varepsilon$-perturbed QCQP (7) have the same feasible set since only the objective function is perturbed. Similarly, their SDP relaxations have the same feasible set. As the feasible sets of QCQP (44) and its SDP relaxation are bounded by Assumption 3.1, the feasible sets for the perturbed problems (7) and (8) are also bounded. The $\varepsilon$-perturbed problem (7) will be used to check whether the SDP relaxation of (4) is exact.

In the following lemma, we show that the exactness of the SDP relaxation for the original problem (4) can be determined by that of perturbed problems (7) and (8).

Lemma 3.3. Let $P \neq O$ be an $n \times n$ nonzero matrix, and $\left\{\varepsilon_{t}\right\}_{t=1}^{\infty}$ be a sequence such that $\lim _{t \rightarrow \infty} \varepsilon_{t}=0$. If the SDP relaxation of the $\varepsilon_{t}$-perturbed problem (7) is exact for all $t=1,2, \ldots$, then the original problem (41) also has an exact SDP relaxation.

Proof. Let $\Gamma$ and $\Delta$ be the feasible sets of (4) and (5), respectively:

$$
\begin{aligned}
\Gamma & :=\left\{x \in \mathbb{R}^{n} \mid Q^{p} \bullet\left(x x^{\mathrm{T}}\right) \leq b_{p}, \quad p=1, \ldots, m\right\}, \\
\Delta & :=\left\{X \in \mathbb{S}^{n} \mid X \succeq O, Q^{p} \bullet X \leq b_{p}, \quad p=1, \ldots, m\right\} .
\end{aligned}
$$

Note that $\Gamma$ is a closed set and both $\Gamma$ and $\Delta$ are bounded by Assumption 3.1. Thus, $\Gamma$ is a compact set in $\mathbb{R}^{n}$. For any $t \geq 1$, let $x_{t}$ and $X_{t}$ be optimal solutions of (7) and (8) satisfying $x_{t} x_{t}^{\mathrm{T}}=X_{t}$, which follows from the assumption on the exactness of the relaxation of (7). As a result, a sequence as $\left\{x_{t}\right\}_{t=1}^{\infty}$ can be defined.

Since the feasible sets of (41) and (77) are identical, we have $x_{t} \in \Gamma$. From the compactness of $\Gamma$, there exists $x_{\lim }:=\lim _{t \rightarrow \infty} x_{t}$ in $\Gamma$. As $X_{\lim }:=x_{\lim } x_{\lim }{ }^{\mathrm{T}} \in \Delta$ by the relationship between $\Gamma$ and $\Delta$, the rank-1 matrix $X_{\text {lim }}$ is also feasible for (51). 
To show that $X_{\text {lim }}$ is an optimal solution of (5), we assume that there exists another feasible $X_{\mathrm{opt}} \neq X_{\text {lim }}$ such that $\nu:=Q^{0} \bullet X_{\text {lim }}-Q^{0} \bullet X_{\mathrm{opt}}>0$. Since $\Delta$ is bounded, there exists $\mu$ such that $\|X\|_{\max }<\mu$ for any $X \in \Delta$, which implies $\left\|x x^{\mathrm{T}}\right\|_{\max }<\mu$ for any $x \in \Gamma$. For a sufficiently large $t$ satisfying

$$
\varepsilon_{t}<\frac{\nu}{4 n^{2} \mu\|P\|_{\max }} \quad \text { and } \quad\left\|x_{t} x_{t}^{\mathrm{T}}-X_{\lim }\right\|_{\max }<\frac{\nu}{2 n^{2}\left\|Q^{0}\right\|_{\max }}
$$

we have

$$
\begin{aligned}
& Q^{0} \bullet\left(x_{t} x_{t}^{\mathrm{T}}-X_{\mathrm{lim}}\right)>-\frac{\nu}{2}, \\
& \varepsilon_{t} P \bullet\left(x_{t} x_{t}^{\mathrm{T}}\right)>-\frac{\nu}{4}, \\
& Q^{0} \bullet X_{\mathrm{lim}}=Q^{0} \bullet X_{\mathrm{opt}}+\nu, \\
& \frac{\nu}{4}>\varepsilon_{t} P \bullet X_{\mathrm{opt}} .
\end{aligned}
$$

Consequently, adding these inequalities and the equality,

$$
\left(Q^{0}+\varepsilon_{t} P\right) \bullet\left(x_{t} x_{t}^{\mathrm{T}}\right)>\left(Q^{0}+\varepsilon_{t} P\right) \bullet X_{\mathrm{opt}},
$$

which contradicts the optimality of $x_{t} x_{t}{ }^{\mathrm{T}}$ in (8) . The desired result follows.

Zhou et al. [35, Lemma 1] focused on a specific QCQP arising from the oprimal power flow problem, and proved that the exactness of its SDP relaxation can be determined by the SDP relaxation of its $\varepsilon$-perturbed problems. Lemma 3.3 is valid for a slightly more general case, which requires only Assumptions 3.1 and 3.2 .

\subsection{Main results}

We present our main results on the exactness of SDP relaxations for forest-structured QCQPs.

For any fixed indices $k, \ell \in[n]$, we define the system $\left[\mathcal{F}_{k \ell}\right)$ :

$$
y \geq 0, \quad S(y) \succeq O, \quad[S(y)]_{k \ell}=0,
$$

where $[S(y)]_{k \ell}$ represents the $(k, \ell)$-th element of $S(y)$ defined in (6). Since $k, \ell \in[n]$, we can construct $n^{2}$ systems $\left(\overline{\mathcal{F}_{k \ell}}\right)$.

If for some $\bar{k}$ and $\bar{\ell}(1 \leq \bar{k}, \bar{\ell} \leq n)$ and for a feasible point $y$ of (하 $)$ the system $\left(\mathcal{F}_{\bar{k} \bar{\ell}}\right)$ is infeasible, then the value $[S(y)]_{\bar{k} \bar{\ell}}$ must be nonzero.

Theorem 3.4. Suppose (4) is a forest-structured $Q C Q P$. If $\left(\mathcal{F}_{k \ell}\right)$ have no feasible solutions for all $(k, \ell) \in \operatorname{od}(n)$, then the $S D P$ relaxation (5) is exact.

To prove the main result described in Theorem 3.4, we impose a condition in Lemma 3.5 for the exact SDP relaxation: the aggregate sparsity graph should be connected and $\left(\mathcal{F}_{k \ell}\right)$ should have no solutions for any $(k, \ell) \in \operatorname{od}(n)$. We note that the system $\left(\mathcal{F}_{k \ell}\right)$ must be tested for feasibility for all $(k, \ell) \in \operatorname{od}(n)$ in Theorem 3.4. We need additionally to examine whether the aggregate sparsity graph is connected in Lemma 3.5. Thus, the sufficient condition presented in Theorem 3.4 can be applied to more general QCQPs than the one in Lemma 3.5. Lemma 3.5 is followed by a proof for Theorem 3.4 by relaxing the sufficient condition in Lemma 3.5. 
Lemma 3.5. Suppose (44) is a forest-structured $Q C Q P$ and the aggregate sparsity graph $G(\mathcal{V}, \mathcal{E})$ of its $S D P$ relaxation defined in (3) is connected. If $\left(\mathcal{F}_{k \ell}\right)$ have no feasible solutions for all $(k, \ell) \in$ $\operatorname{od}(n)$, then the SDP relaxation (5) is exact.

Proof. Let $X^{*}$ be any optimal solution for (5). By Assumption 3.1, there exists an optimal solution $y^{*}$ for (6). Since $y^{*} \geq 0$ and $S\left(y^{*}\right) \succeq O$, we have $S\left(y^{*}\right)_{k \ell} \neq 0$ for every $(k, \ell) \in \operatorname{od}(n)$ by the assumption. This implies that all the off-diagonal elements on od $(n)$ of the forest-structured matrix $S\left(y^{*}\right)$ are nonzeros, thus $\operatorname{rank} S\left(y^{*}\right) \geq n-1$ by Lemma 2.4. Since Assumption 3.1 (b) and (c) hold, $X^{*} S\left(y^{*}\right)=O$ by the strong duality. From the Sylvester's rank inequality [3], $\operatorname{rank} X^{*}+\operatorname{rank} S\left(y^{*}\right) \leq n+\operatorname{rank} X^{*} S\left(y^{*}\right)$ holds for $X^{*}$ and $S\left(y^{*}\right)$. Therefore, $\operatorname{rank} X^{*} \leq n+$ $\operatorname{rank} O-\operatorname{rank} S\left(y^{*}\right) \leq n+0-(n-1)=1$.

Proof. (Theorem 3.4)

Let $G(\mathcal{V}, \mathcal{E})$ be the aggregate sparsity graph of the SDP relaxation defined in (3) for the foreststructured QCQP, and let $\kappa$ denote the number of connected components of $G(\mathcal{V}, \mathcal{E})$. Since $G(\mathcal{V}, \mathcal{E})$ consists of one or more trees, we can construct a set $\mathcal{D}$ with $\kappa-1$ edges such that $G(\mathcal{V}, \mathcal{E} \cup \mathcal{D})$ is a tree (i.e., a connected graph with no cycles). Let $P:=\sum_{(i, j) \in \mathcal{D}}\left(E_{i j}+E_{j i}\right)$ be a perturbation matrix with the $n \times n$ matrices $E_{i j}$ 's. With $\varepsilon>0$, consider the $\varepsilon$-perturbed QCQP (7) with this $P$. Obviously, the aggregate sparsity graph of the $\varepsilon$-perturbed QCQP (7D) is $G(\mathcal{V}, \mathcal{E} \cup \mathcal{D})$. The system $\left(\mathcal{F}_{k \ell}\right)$ that corresponds to the $\varepsilon$-perturbed QCQP (7) can be written as:

$$
\begin{gathered}
y \geq 0, \quad Q^{0}+\sum_{(i, j) \in \mathcal{D}} \varepsilon\left(E_{i j}+E_{j i}\right)+\sum_{p=1}^{m} y_{p} Q^{p} \succeq O, \\
{\left[Q^{0}+\sum_{(i, j) \in \mathcal{D}} \varepsilon\left(E_{i j}+E_{j i}\right)\right]_{k \ell}+\sum_{p=1}^{m} y_{p}\left[Q^{p}\right]_{k \ell}=0 .}
\end{gathered}
$$

For the exact SDP relaxation of (7), we need to show that (9) has no feasible solutions for all $(k, \ell) \in \operatorname{od}(n) \cup \mathcal{D}$. First, suppose $(k, \ell) \in \mathcal{D}$. Since $\left[Q^{p}\right]_{k \ell}=0(\forall p=0,1, \ldots, m)$, the left hand side of (9b) becomes

$$
\varepsilon \sum_{(i, j) \in \mathcal{D}}\left[E_{i j}+E_{j i}\right]_{k \ell}=\varepsilon>0 .
$$

We have shown that (9b) does not hold for any $y \geq 0$.

Next, we suppose $(k, \ell) \in \operatorname{od}(n)$. Assume that (9) has a solution $\hat{y}$. Then,

$$
\hat{y} \geq 0, \quad Q^{0}+\sum_{(i, j) \in \mathcal{D}} \varepsilon\left(E_{i j}+E_{j i}\right)+\sum_{p=1}^{m} \hat{y}_{p} Q^{p} \succeq O, \quad\left[Q^{0}\right]_{k \ell}+\sum_{p=1}^{m} \hat{y}_{p}\left[Q^{p}\right]_{k \ell}=0 .
$$

We now define a forest-structured matrix $\bar{S} \in \mathbb{S}^{n}$ as:

$$
[\bar{S}]_{q r}= \begin{cases}{\left[Q^{0}+\sum_{(i, j) \in \mathcal{D}} \varepsilon\left(E_{i j}+E_{j i}\right)+\sum_{p=1}^{m} \hat{y}_{p} Q^{p}\right]_{q r}} & (q, r) \notin \mathcal{D}, \\ 0 & (q, r) \in \mathcal{D} .\end{cases}
$$

If $(q, r) \notin \mathcal{D}$, by definition, we have $\left[E_{i j}+E_{j i}\right]_{q r}=0$ for any $(i, j) \in \mathcal{D}$. If $(q, r) \in \mathcal{D}$, we have $\left[Q^{0}+\sum_{p=1}^{m} \hat{y}_{p} Q^{p}\right]_{q r}=0$ since $\left[Q^{p}\right]_{q r}=0$ for any $p \in\{0\} \cup[m]$. Thus, we obtain that 
$\bar{S}=Q^{0}+\sum_{p=1}^{m} \hat{y}_{p} Q^{p}$. By Lemma 2.5, it follows that $\bar{S} \succeq O$, which implies that $\hat{y}$ solves the following system:

$$
y \geq 0, \quad Q^{0}+\sum_{p=1}^{m} y_{p} Q^{p} \succeq O, \quad\left[Q^{0}\right]_{k \ell}+\sum_{p=1}^{m} y_{p}\left[Q^{p}\right]_{k \ell}=0 .
$$

We know that the system (10), which is equivalent to $\left(\mathcal{F}_{k \ell}\right)$ for (44), has no feasible points by the assumption. This is a contradiction. Thus, (9) has no feasible points. By Lemma 3.5, the SDP relaxation for (17) is exact for all $\varepsilon>0$.

We now take a sequence $\left\{\varepsilon_{t}\right\}_{t=1}^{\infty}$ which converges to zero so that the SDP relaxation for $\varepsilon_{t^{-}}$ perturbed QCQP (7) is exact for all $t=1,2, \ldots$ By Lemma 3.3, we conclude that the SDP relaxation for (4) is exact.

Theorem 3.4 can be applied to the particular case of tridiagonal QCQPs that will be discussed in section 4 .

Since $|\operatorname{od}(n)| \leq n-1$ by definition, we must solve at most $n-1$ systems in order to determine whether the SDP relaxation of a QCQP is exact. It may be very time-consuming to solve all $n-1$ systems $\left(\mathcal{F}_{k \ell}\right)$ due to the positive semidefinite constraint. To mitigate this difficulty, conditions that do not dependent on $\left(\mathcal{F}_{k \ell}\right)$ will be discussed in Corollaries 3.6 and 3.7

It was shown in [6, 15, 19] that QCQP (11) can be solved exactly by the SDP relaxation if the data matrices of the QCQP is sign-definite. Using the feasibility of the system $\left(\overrightarrow{\mathcal{F}_{k \ell}}\right)$, we provide an alternative proof for the exact SDP relaxation of the QCQP (4) if the set of $(i, j)$-th elements of all matrices is sign-definite for every superdiagonal index $(i, j)$.

Corollary 3.6. Suppose (44) is a forest-structured $Q C Q P$. If the set $\left\{\left[Q^{0}\right]_{k \ell},\left[Q^{1}\right]_{k \ell}, \ldots,\left[Q^{m}\right]_{k \ell}\right\}$ is sign-definite for all $(k, \ell) \in \operatorname{od}(n)$, then the $S D P$ relaxation (5) is exact.

Proof. Define $P:=\left[p_{i j}\right] \in \mathbb{S}^{n}$ where

$$
p_{i j}:= \begin{cases}+1 & \text { if }\left[Q^{0}\right]_{i j}=0 \text { and } \sum_{p=1}^{m}\left[Q^{p}\right]_{i j} \geq 0 \\ -1 & \text { if }\left[Q^{0}\right]_{i j}=0 \text { and } \sum_{p=1}^{m}\left[Q^{p}\right]_{i j}<0 \\ 0 & \text { otherwise }\end{cases}
$$

Let $\left\{\varepsilon_{t}\right\}_{t=0}^{\infty} \subset \mathbb{R}$ be a sequence converging to 0 . Consider the $\varepsilon_{t}$-perturbed problem (77) with the $P$ defined above. The corresponding system $\left(\overrightarrow{\mathcal{F}_{k \ell}}\right)$ is

$$
y \geq 0, \quad S(y)=Q^{0}+\varepsilon_{t} P+\sum_{p=1}^{m} y_{p} Q^{p} \succeq O, \quad[S(y)]_{k \ell}=\left[Q^{0}\right]_{k \ell}+\varepsilon_{t} p_{k \ell}+\sum_{p=1}^{m} y_{p}\left[Q^{p}\right]_{k \ell}=0 .
$$

Now we analyze the feasibility of the system for any $(k, \ell) \in \operatorname{od}(n)$.

(a) If $\left[Q^{0}\right]_{k \ell} \neq 0$, then

$$
\left[Q^{0}\right]_{k \ell}+\sum_{p=1}^{m} y_{p}\left[Q^{p}\right]_{k \ell}= \begin{cases}>0 & \text { if }\left[Q^{0}\right]_{k \ell}>0 \\ <0 & \text { if }\left[Q^{0}\right]_{k \ell}<0\end{cases}
$$

by the sign-definite assumption on the set $\left\{\left[Q^{0}\right]_{k \ell},\left[Q^{1}\right]_{k \ell}, \ldots,\left[Q^{m}\right]_{k \ell}\right\}$. We have $[S(y)]_{k \ell} \neq 0$ for any $y \geq 0$ from $p_{k \ell}=0$, therefore the system has no solution. 
(b) If $\left[Q^{0}\right]_{k \ell}=0$, then for any $y \geq 0$,

$$
\left[Q^{0}\right]_{k \ell}+\sum_{p=1}^{m} y_{p}\left[Q^{p}\right]_{k \ell}= \begin{cases}\geq 0 & \text { if } \sum_{p=1}^{m}\left[Q^{p}\right]_{k \ell} \geq 0 \\ \leq 0 & \text { otherwise }\end{cases}
$$

From

$$
\varepsilon_{t} p_{k \ell}= \begin{cases}\varepsilon>0 & \text { if } \sum_{p=1}^{m}\left[Q^{p}\right]_{k \ell} \geq 0, \\ -\varepsilon<0 & \text { otherwise, }\end{cases}
$$

we have $[S(y)]_{k \ell} \neq 0$, which implies that the system has no solution.

As (a) and (b) cover all possible cases, the SDP relaxation of the $\varepsilon_{t}$-perturbed problem is exact for any $\varepsilon_{t}$ by Theorem 3.4 hence the original QCQP (4) is also exact by Lemma 3.3.

We will use Corollary 3.6 to prove the exact SDP relaxation for a class of QCQPs in subsection 4.2.

Next, we examine the following QCQP:

$$
\begin{array}{ll}
\operatorname{minimize} & x^{\mathrm{T}} Q^{0} x \\
\text { subject to } & x^{\mathrm{T}} Q^{1} x=b_{p} .
\end{array}
$$

To write (11) in the form of (4), the equality constraint is converted into two inequality constraints $x^{\mathrm{T}} Q^{1} x \leq b_{1}$ and $x^{\mathrm{T}}\left(-Q^{1}\right) x \leq-b_{1}$. It is clear that problem (11) is a special case of QCQP (4) with two constraints. Since both matrices $Q^{1}$ and $-Q^{1}$ appear in the inequality constraints of (11), the set $\left\{\left[Q^{0}\right]_{i j},\left[Q^{1}\right]_{i j},-\left[Q^{1}\right]_{i j}\right\}$ is not sign-definite unless $\left[Q^{1}\right]_{i j}=0$. As a result, Corollary 3.6 cannot be used to determine the exactness of the SDP relaxation of (4). For (11), we propose the following exactness condition.

Corollary 3.7. Suppose (4) is a forest-structured $Q C Q P$. If for all $(i, j) \in \operatorname{od}(n)$ such that $\left[Q^{1}\right]_{i j} \neq$ 0 ,

$$
Q^{0}-\frac{\left[Q^{0}\right]_{i j}}{\left[Q^{1}\right]_{i j}} Q^{1} \nsucceq O,
$$

then the SDP relaxation for (11) is exact.

Proof. Let $\mathcal{E}$ be the aggregate sparsity structure of the SDP relaxation defined in subsection 2.3. If $\left(\mathcal{F}_{k \ell}\right)$ are infeasible for all $(k, \ell) \in \operatorname{od}(n)$, then we know from Theorem 3.4 that the SDP relaxation is exact. Thus, we analyze the feasibility of the system $\left(\mathcal{F}_{k \ell}\right)$ for each $(k, \ell) \in \operatorname{od}(n)$. We compute $S(y)$ in the system as:

$$
\begin{aligned}
S(y) & =Q^{0}+y_{1} Q^{1}+y_{2}\left(-Q^{1}\right), \quad y=\left[\begin{array}{l}
y_{1} \\
y_{2}
\end{array}\right] \geq 0, \\
& =Q^{0}+z Q^{1}, \quad z:=y_{1}-y_{2} \in \mathbb{R} .
\end{aligned}
$$

Then, the system $\left(\mathcal{F}_{k \ell}\right)$ is equivalent to the following system:

$$
Q^{0}+z Q^{1} \succeq O, \quad\left[Q^{0}\right]_{k \ell}+z\left[Q^{1}\right]_{k \ell}=0 .
$$

(a) If $\left[Q^{1}\right]_{k \ell}=0$, then $\left[Q^{0}\right]_{k \ell} \neq 0$ by $(k, \ell) \in \operatorname{od}(n)$. Since any $z \in \mathbb{R}$ does not satisfy the second equation in (12), the system (12) is infeasible. 
(b) If $\left[Q^{1}\right]_{k \ell} \neq 0$, we assume that a solution $z^{*}$ to (12) exists. By solving the second equation in (12) for $z^{*}$ and substituting it into the first equation, we have

$$
Q^{0}-\frac{\left[Q^{0}\right]_{i j}}{\left[Q^{1}\right]_{i j}} Q^{1} \succeq O
$$

which is a contradiction. Thus, the desired result follows.

Burer and Ye [7] proposed several methods to extend their result of diagonal QCQPs to a general class of QCQPs with exact SDP relaxations. However, for some QCQPs, it is difficult to show the exactness of relaxations by their conditions; for example, it is hard to prove the existence of an exact relaxation for the generalized trust-region subproblem (see section 4).

\section{Extension to a wider class of QCQPs based on simultaneous tridiagonalization}

To apply the results in section 3 to a wider class of QCQPs, we consider QCQPs where the data matrices in (11) are not forest-structured. In this section, we suppose Assumption 3.1 (a) - (c) and that all the matrices of a QCQP are simultaneously tridiagonalizable. In section 4.1, simultaneous tridiagonalization is discussed in detail when $m=1$ (only one quadratic constraint). Then, a method to determine the exactness of the SDP relaxation for these QCQPs is described. In addition, we provide an alternative proof for the exactness of Generalized Trust-Region Subproblems (GTRS).

The matrices $Q^{0}, Q^{1}, \ldots, Q^{m} \in \mathbb{S}^{n}$ are called simultaneous tridiagonalizable if there exist a nonsingular matrix $U$ such that $U^{\mathrm{T}} Q^{0} U, U^{\mathrm{T}} Q^{1} U, \ldots, U^{\mathrm{T}} Q^{m} U$ are tridiagonal matrices. The simultaneous tridiagonalization is a generalization of the simultaneous diagonalization used in [7]. By replacing $U^{-1} x$ with $\hat{x}$, we obtain the tridiagonal QCQP equivalent to (1):

$$
\begin{array}{ll}
\operatorname{minimize} & \hat{x}^{\mathrm{T}}\left(U^{\mathrm{T}} Q^{0} U\right) \hat{x}+2\left(U^{\mathrm{T}} q_{0}\right)^{\mathrm{T}} \hat{x} \\
\text { subject to } & \hat{x}^{\mathrm{T}}\left(U^{\mathrm{T}} Q^{p} U\right) \hat{x}+2\left(U^{\mathrm{T}} q_{p}\right)^{\mathrm{T}} \hat{x} \leq b_{p}, \quad p=1, \ldots, m .
\end{array}
$$

The standard SDP relaxation of (13) is

$$
\begin{array}{ll}
\operatorname{minimize} & \left(U^{\mathrm{T}} Q^{0} U\right) \bullet \hat{X}+2\left(U^{\mathrm{T}} q_{0}\right)^{\mathrm{T}} \hat{x} \\
\text { subject to } & \left(U^{\mathrm{T}} Q^{p} U\right) \bullet \widehat{X}+2\left(U^{\mathrm{T}} q_{p}\right)^{\mathrm{T}} \hat{x} \leq b_{p}, \quad p=1, \ldots, m, \\
& \widehat{X} \succeq \hat{x} \hat{x}^{\mathrm{T}} .
\end{array}
$$

Obviously, if $\hat{x}$ is an optimal solution of (13), then $U \hat{x}$ is an optimal solution of the original problem (11). We notice that the SDP relaxation (2) of (11) is at least as strong as the corresponding SDP relaxation (14) for (13). As a result, if (13) has an exact relaxation, then (1) also has an exact relaxation. When (13) becomes a forest-structured QCQP in the homogeneous form, the exactness conditions in section 3 can be applied to (13). We note that the exactness of the SDP relaxation for QCQPs (11) can be determined if their data matrices are simultaneous tridiagonalizable even when they are not forest-structured. 


\subsection{Simultaneous tridiagonalization}

Simultaneous tridiagonalization of multiple matrices is an extension of simultaneous diagonalization, and it can be achieved by finding a nonsingular matrix that transforms all matrices to tridiagonal matrices. Recently, Sidje [29] (See also Garvey et al. [10]) introduced conditions under which two matrices are simultaneous tridiagonalizable.

Proposition 4.1. [29] Let $K, M \in \mathbb{S}^{n}$ and $0 \neq \gamma \in \mathbb{R}$. Suppose that the matrix pencil $K-\gamma M$ is nonsingular. Then, $K$ and $M$ are simultaneously tridiagonalizable.

If the data matrices are simultaneously tridiagonalizable with the nonsingular matrix $U$, we also need a method to compute $U$. Sidje proposed a method to compute such a nonsingular matrix $U$ on the basis of Householder reflections. We briefly describe his method for the simultaneous tridiagonalization, and then analyze this method to find new properties which will be used in the proof of the GTRS.

In the beginning of the Sidje's recursive procedure [29], the matrices are initialized as $K^{n}:=K$, $M^{n}:=M \in \mathbb{S}^{n}$. Then, an appropriate nonsingular matrix $U^{k}=\left[1,0_{k-1}{ }^{\mathrm{T}} ; u_{k}, \widetilde{U}^{k}\right] \in \mathbb{R}^{k \times k}$ with nonsingular $\widetilde{U}^{k} \in \mathbb{R}^{(k-1) \times(k-1)}$ and $u_{k} \in \mathbb{R}^{k-1}$ is chosen such that

$$
\left(U^{k}\right)^{\mathrm{T}} K^{k} U^{k}=\left[\begin{array}{c:c}
\xi_{k} & \tau_{k} 0_{k-2}^{\mathrm{T}} \\
\hdashline \tau_{k}- & K^{k-1} \\
0_{k-2} &
\end{array}\right], \quad\left(U^{k}\right)^{\mathrm{T}} M^{k} U^{k}=\left[\begin{array}{c:cc}
\nu_{k} & \sigma_{k} 0_{k-2}{ }^{\mathrm{T}} \\
\hdashline \sigma_{k} & M^{k-1} \\
0_{k-2} &
\end{array}\right]
$$

at each step $k=n, \ldots, 2$. Here $\xi_{k}, \tau_{k}, \nu_{k}, \sigma_{k} \in \mathbb{R}$, and $K^{k-1}, M^{k-1} \in \mathbb{R}^{(k-1) \times(k-1)}$. This procedure generates two tridiagonal matrices:

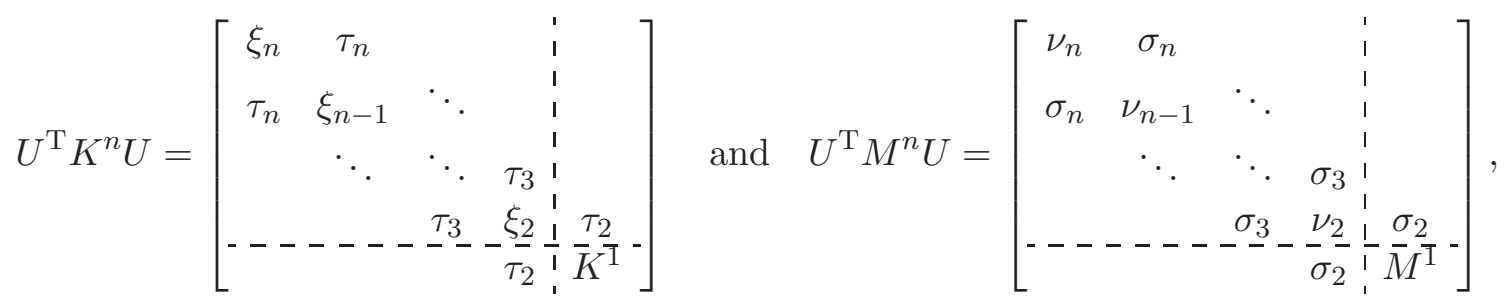

where

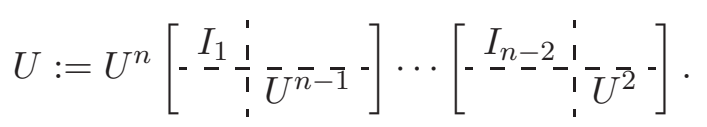

Now, consider the step $k$. To have nonzero elements only on the diagonals, superdiagonals, and subdiagonals by the operation shown in (15), $\widetilde{U}^{k}$ should satisfy the following equation:

$$
\left(\widetilde{U}^{k}\right)^{\mathrm{T}}\left(K_{\{1\},\{2, \ldots, k\}}^{k}+K_{\{2, \ldots, k\}}^{k} u_{k}\right)=\tau_{k} e_{1}, \quad\left(\widetilde{U}^{k}\right)^{\mathrm{T}}\left(M_{\{1\},\{2, \ldots, k\}}^{k}+M_{\{2, \ldots, k\}}^{k} u_{k}\right)=\sigma_{k} e_{1},
$$

where $\boldsymbol{e}_{1} \in \mathbb{R}^{k-1}$ and $K_{\{2, \ldots, k\}}^{k}$ means the submatrix of $K^{k}$ obtained by removing the first row and column from $K^{k}$, as mentioned in section 2.1. In his procedure, $\widetilde{U}^{k}$ is chosen to be a Householder reflection, and therefore, nonsingular, and the existence of $u_{k}$ follows imposing

$$
\left(\widetilde{U}^{k}\right)^{\mathrm{T}}\left(K_{\{1\},\{2, \ldots, k\}}^{k}+K_{\{2, \ldots, k\}}^{k} u_{k}\right)=\gamma\left(\widetilde{U}^{k}\right)^{\mathrm{T}}\left(M_{\{1\},\{2, \ldots, k\}}^{k}+M_{\{2, \ldots, k\}}^{k} u_{k}\right)
$$

for $0 \neq \gamma \in \mathbb{R}$ since $K-\gamma M$ is nonsingular. By restricting $\gamma$ to be positive, we extend Proposition 4.1. 
Lemma 4.2. Let $K, M \in \mathbb{S}^{n}$ and $\mathbb{R} \ni \gamma>0$. Suppose that the matrix pencil $K-\gamma M$ is nonsingular. Then, there exists a nonsingular matrix $U \in \mathbb{S}^{n}$ that simultaneously tridiagonalizes $K$ and $M$. Moreover, for any $i \in[n-1]$, the $(i, i+1)$-th elements of $U^{\mathrm{T}} K U$ and $U^{\mathrm{T}} M U$ are sign-definite.

Proof. Substituting (16) into (17), we obtain $\tau_{k}=\gamma \sigma_{k}$. Since $\gamma>0$, the set $\left\{\tau_{k}, \sigma_{k}\right\}$ is sign-definite for any $k=n, \ldots, 2$. The variables $\tau_{k}$ and $\sigma_{k}$ appear on the $(1,2), \ldots,(n-1, n)$-th elements of $U^{\mathrm{T}} K^{n} U$ and $U^{\mathrm{T}} M^{n} U$, respectively.

If the elements on superdiagonal of all matrices can be transformed to sign-definite elements, then we can apply Corollary 3.6 to show the exactness of the SDP relaxation, which is discussed next in subsection 4.2 .

\subsection{Generalized trust-region subproblem}

Consider the Generalized Trust-Region Subproblem (GTRS):

$$
\begin{array}{ll}
\text { minimize } & x^{\mathrm{T}} Q^{0} x+2 q_{0}{ }^{\mathrm{T}} x \\
\text { subject to } & x^{\mathrm{T}} Q^{1} x+2 q_{1}{ }^{\mathrm{T}} x \leq b_{1} .
\end{array}
$$

The GTRS can be considered a generalization of the classical TRS that minimizes a quadratic objective over an Euclidean ball, i.e., the GTRS with $Q^{1} \succ O$. This fact can be seen by substituting $\sqrt{Q^{1}} x$ as a new variable $\tilde{x}$, where $\sqrt{Q^{1}}$ denotes the Cholesky factor of $Q^{1}$, i.e., $Q^{1}={\sqrt{Q^{1}}}^{\mathrm{T}} \sqrt{Q^{1}}$. In the TRS, $Q^{0}$ is not necessarily positive definite. Although the TRS is nonlinear and nonconvex, its SDP relaxation is well-known to be exact. The GTRS shares nice properties with the TRS. For example, by using S-lemma, it is proved that the SDP relaxation of the GTRS is always exact under the Slater's condition.

Since the GTRS is clearly a QCQP (11) with only one constraint $(m=1)$, we can also prove that the GTRS admits an exact SDP relaxation with the exactness conditions presented in section 3 . Although the result is certainly not new, our proof shows a procedure on how to apply the exactness conditions for tridiagonal QCQPs to wider classes of QCQPs. In fact, the proof demonstrates how to determine the exactness of a given QCQP in practice, and it can be used to analyze the exactness conditions for broader classes of QCQPs.

Any QCQP can be formulated in the equivalent homogeneous QCQP as in (44), thus, it is sufficient to consider the following QCQP with an additional variable to discuss the exactness for the GTRS (18):

$$
\begin{array}{ll}
\operatorname{minimize} & x^{\mathrm{T}} \bar{Q}^{0} x \\
\text { subject to } & x^{\mathrm{T}} \bar{Q}^{1} x \leq 0 \\
& x^{\mathrm{T}} E_{11} x=1
\end{array}
$$

where

$$
\bar{Q}^{p}:=\left[\begin{array}{cc}
-b_{p} & q_{p}^{\mathrm{T}} \\
q_{p} & Q^{p}
\end{array}\right] \quad(p=0,1) \quad \text { and } \quad b_{0}=0
$$

For simplicity, we assume that the number of variables in (19) is $n$. As (19) has the additional equality constraint, the problem (19) is a QCQP with three inequality constraints, and (19) is no longer a GTRS. In the subsequent discussion, we describe the exactness for (19) using the simultaneous tridiagonalization. In particular, we show that the SDP relaxation of GTRS (18) is exact as follows. 
Theorem 4.3. Suppose that GTRS (19) satisfies Assumption 3.1. Then, the SDP relaxation of (19) is exact.

Proof. Let us first consider the case when $\bar{Q}^{0}-\gamma \bar{Q}^{1}$ is nonsingular for some $\gamma>0$. By Lemma 4.2. we obtain a nonsingular matrix $U \in \mathbb{S}^{n}$ that simultaneously tridiagonalizes $Q^{0}$ and $Q^{1}$, and the $(i, i+1)$-th elements of $U^{\mathrm{T}} \bar{Q}^{0} U$ and $U^{\mathrm{T}} \bar{Q}^{1} U$ become sign-definite for any $i \in[n-1]$. Call these tridiagonal matrices

$$
R^{p}=\left[r_{i j}^{p}\right]:=U^{\mathrm{T}} \bar{Q}^{p} U, \quad p=0,1 .
$$

For any $i \in[n-1]$, the set $\left\{r_{i, i+1}^{0}, r_{i, i+1}^{1}\right\}$ is sign-definite. By letting $y=U^{-1} x$, the homogeneous TRS (19) can be transformed to an equivalent tridiagonal QCQP:

$$
\begin{array}{ll}
\text { minimize } & y^{\mathrm{T}} R^{0} y \\
\text { subject to } & y^{\mathrm{T}} R^{1} y \leq 0 \\
& y^{\mathrm{T}} E_{11} y=1 .
\end{array}
$$

Notice that the first row of $U$ is $\left[10_{n-1}^{\mathrm{T}}\right]$ by the construction of section 4.1. By Corollary 3.6, the SDP relaxation of (20) is exact. As $U$ is nonsingular, the SDP relaxation of the original problem (19) is also exact.

Now consider the other case, i.e., there is no $\gamma>0$ such that $\bar{Q}^{0}-\gamma \bar{Q}^{1}$ is nonsingular. We will show that, for a fixed $\gamma>0$ and any $\varepsilon>0$, the SDP relaxation of the following $\varepsilon$-perturbed problem is exact:

$$
\begin{array}{cl}
\operatorname{minimize} & x^{\mathrm{T}}\left(\bar{Q}^{0}+\varepsilon I_{n}\right) x \\
\text { subject to } & x^{\mathrm{T}} \bar{Q}^{1} x \leq 0 \\
& x^{\mathrm{T}} E_{11} x=1 .
\end{array}
$$

Then, by Lemma 3.3. the SDP relaxation of the original problem (19) is also exact. Since $\operatorname{det}\left(\bar{Q}^{0}-\gamma \bar{Q}^{1}\right)=0, \bar{Q}^{0}-\gamma \bar{Q}^{1}$ can be diagonalized, as below:

$$
\left[\begin{array}{cc}
\Lambda & \\
& O_{n-\mathrm{rk}}
\end{array}\right]=P^{\mathrm{T}}\left(\bar{Q}^{0}-\gamma \bar{Q}^{1}\right) P,
$$

where rk $:=\operatorname{rank}\left(\bar{Q}^{0}-\gamma \bar{Q}^{1}\right), P \in \mathbb{R}^{n \times n}$ is an orthogonal matrix, and $\Lambda \in \mathbb{S}^{\text {rk }}$ is a diagonal matrix. By adding perturbation with sufficiently small $\varepsilon>0$ to both sides of (22),

$$
\left[\begin{array}{cc}
\Lambda & \\
& O_{n-\mathrm{rk}}
\end{array}\right]+\varepsilon I_{n}=P^{\mathrm{T}}\left(\bar{Q}^{0}-\gamma \bar{Q}^{1}\right) P+\varepsilon P^{\mathrm{T}} I_{n} P=P^{\mathrm{T}}\left(\bar{Q}^{0}+\varepsilon I_{n}-\gamma \bar{Q}^{1}\right) P
$$

$\left(\bar{Q}^{0}+\varepsilon I_{n}\right)-\gamma \bar{Q}^{1}$ becomes nonsingular. From the first case of this proof, the SDP relaxation of (21) must be exact.

\section{Concluding remarks}

We have presented sufficient conditions for the SDP relaxation of a class of QCQPs to be exact by investigating the rank of the aggregated sparsity matrix of QCQPs. The class of QCQPs is forest-structured QCQPs that include tridiagonal QCQPs, arrow-type QCQPs, and QCQPs with simultaneously tridiagonalizable data matrices. The signs of data matrix elements have not been used to determining the exactness of the SDP relaxation. 
In our proof for the main results, we have utilized the fact that any symmetric positive semidefinite matrix whose graph is a tree has rank at least $n-1$. It is shown in [13] that for any non-tree the minimum rank of a positive semidefinite matrix is less than $n-1$.

We have also extended our results to non-tridiagonal QCQPs by improving a computing method proposed in 29] for simultaneous tridiagonalization. The exactness of the SDP relaxation of the GTRS can be proved by the simultaneous tridiagonalization and our results in section 3 .

For a future work, we want to extend the result to a wider class of QCQPs. Algorithms for simultaneous tridiagonalization of multiple matrices will be further studied to apply our results.

\section{References}

[1] S. Adachi and Y. Nakatsukasa. Eigenvalue-based algorithm and analysis for nonconvex qcqp with one constraint. Mathematical Programming, 173(1):79-116, 2019.

[2] M. F. Anjos and J. B. Lasserre, editors. Handbook on Semidefinite, Conic and Polynomial Optimization, volume 166 of International Series in Operations Research 8 Management Science. Springer, New York, NY 10013, USA, 2012.

[3] H. Anton and C. Rorres. Elementary Linear Algebra: Applications Version. John Wiley \& Sons Inc., USA, 11th ed. edition, 2014.

[4] X. Bao, N. V. Sahinidis, and M. Tawarmalani. Semidefinite relaxations for quadratically constrained quadratic programming: A review and comparisons. Mathematical Programming, $129(1): 129,2011$.

[5] P. Biswas, T.-C. Lian, T.-C. Wang, and Y. Ye. Semidefinite programming based algorithms for sensor network localization. ACM Transactions on Sensor Networks, 2(2):188-220, 2006.

[6] S. Bose, D. F. Gayme, K. M. Chandy, and S. H. Low. Quadratically constrained quadratic programs on acyclic graphs with application to power flow. IEEE Transactions on Control of Network Systems, 2(3):278-287, 2015.

[7] S. Burer and Y. Ye. Exact semidefinite formulations for a class of (random and non-random) nonconvex quadratic programs. Mathematical Programming, 181(1):1-17, 2020.

[8] M. El-Mikkawy. A note on a three-term recurrence for a tridiagonal matrix. Applied Mathematics and Computation, 139(2):503-511, 2003.

[9] M. Fukuda, M. Kojima, K. Murota, and K. Nakata. Exploiting sparsity in semidefinite programming via matrix completion I: General framework. SIAM Journal on Optimization, 11(3):647-674, 2001.

[10] S. D. Garvey, F. Tisseur, M. I. Friswell, J. E. T. Penny, and U. Prells. Simultaneous tridiagonalization of two symmetric matrices. International Journal for Numerical Methods in Engineering, 57(12):1643-1660, 2003.

[11] N. Ito, S. Kim, M. Kojima, A. Takeda, and K. Toh. BBCPOP: A sparse doubly nonnegative relaxation of polynomial optimization problems with binary, box and complementarity constraints. ACM Transactions on Mathematical Software, 45(3):34, 2019. 
[12] C. R. Johnson and A. Leal Duarte. The maximum multiplicity of an eigenvalue in a matrix whose graph is a tree. Linear and Multilinear Algebra, 46(1-2):139-144, 1999.

[13] C. R. Johnson and A. Leal-Duarte. Converse to the parter-wiener theorem: The case of non-trees. Discrete Mathematics, 306(23):3125-3129, 2006.

[14] C. R. Johnson, A. Leal Duarte, C. M. Saiago, B. D. Sutton, and A. J. Witt. On the relative position of multiple eigenvalues in the spectrum of an hermitian matrix with a given graph. Linear Algebra and its Applications, 363:147-159, 2003.

[15] S. Kim and M. Kojima. Exact solutions of some nonconvex quadratic optimization problems via SDP and SOCP relaxations. Computational Optimization and Applications, 26(2):143$154,2003$.

[16] S. Kim, M. Kojima, M. Mevissen, and M. Yamashita. Exploiting sparsity in linear and nonlinear matrix inequalities via positive semidefinite matrix completion. Mathematical programming, 129(1):33-68, 2011.

[17] T. C. Koopmans and M. Beckmann. Assignment problems and the location of economic activities. Econometrica, 25(1):53-76, 1957.

[18] M. Laurent and A. Varvitsiotis. A new graph parameter related to bounded rank positive semidefinite matrix completions. Mathematical Programming, 145(1):291-325, 2014.

[19] J. Lavaei and S. H. Low. Zero duality gap in optimal power flow problem. IEEE Transactions on Power Systems, 27(1):92-107, 2012.

[20] R. Madani, S. Sojoudi, G. Fazelnia, and J. Lavaei. Finding low-rank solutions of sparse linear matrix inequalities using convex optimization. SIAM Journal on Optimization, 27(2):725-758, 2017.

[21] K. Nakata, K. Fujisawa, M. Fukuda, M. Kojima, and K. Murota. Exploiting sparsity in semidefinite programming via matrix completion II: Implementation and numerical results. Mathematical Programming, 95(2):303-327, 2003.

[22] Y. Nesterov, H. Wolkowicz, and Y. Ye. Semidefinite Programming Relaxations of Nonconvex Quadratic Optimization, volume 27 of Handbook of Semidefinite Programming. International Series in Operations Research $\& 5$ Management Science, pages 361-419. Springer, Boston, MA, 2000 .

[23] G. Pataki. On the rank of extreme matrices in semidefinite programs and the multiplicity of optimal eigenvalues. Mathematics of Operations Research, 23(2):339-358, 1998.

[24] I. Pólik and T. Terlaky. A survey of the s-lemma. SIAM Review, 49(3):371-418, 2007.

[25] J. Povh and F. Rendl. Copositive and semidefinite relaxations of the quadratic assignment problem. Discrete Optimization, 6(3):231-241, 2009.

[26] S. Safarina, S. Moriguchi, T. J. Mullin, and M. Yamashita. Conic relaxation approaches for equal deployment problems. Discrete Applied Mathematics, 275:111-125, 2020.

[27] S. Safarina, T. J. Mullin, and M. Yamashita. Polyhedral-based methods for mixed-integer socp in tree breeding. Journal of the Operations Research Society of Japan, 62(4):133-151, 2019 . 
[28] H. Sheen and M. Yamashita. Exploiting aggregate sparsity in second order cone relaxations for quadratic constrained quadratic programming problems. arXiv: 1911.02188, 2019.

[29] R. B. Sidje. On the simultaneous tridiagonalization of two symmetric matrices. Numerische Mathematik, 118(3):549-566, 2011.

[30] S. Sojoudi and J. Lavaei. Exactness of semidefinite relaxations for nonlinear optimization problems with underlying graph structure. SIAM Journal on Optimization, 24(4):1746-1778, 2014.

[31] A. L. Wang and F. Kilinç-Karzan. The generalized trust region subproblem: solution complexity and convex hull results. arXiv:1907.08843, 2019.

[32] A. L. Wang and F. Kilinç-Karzan. On the tightness of sdp relaxations of qcqps. Optimization Online, January, 2020.

[33] H. Wolkowicz, R. Saigal, and L. Vandenberghe. Handbook of semidefinite programming: theory, algorithms, and applications. Springer, New York, USA, 2000.

[34] L. Q. Yang, D. F. Sun, and K. C. Toh. SDPNAL+: a majorized semismooth Newton-CG augmented Lagrangian method for semidefinite programming with nonnegative constraints. Mathematical Programming Computation, 7(3):331-366, 2015.

[35] F. Zhou, Y. Chen, and S. H. Low. Sufficient conditions for exact semidefinite relaxation of optimal power flow in unbalanced multiphase radial networks. In IEEE 58th Conference on Decision and Control (CDC), volume 58, pages 6227-6233, 2019. 